\title{
Atomistic Models of Carbonate Minerals: Bulk and Surface Structures, Defects, and Diffusion
}

\author{
Randall T. Cygan ${ }^{1}$, Kate Wright ${ }^{2}$, Diana K. Fisler ${ }^{3}$, Julian D. Gale ${ }^{4}$, and Ben Slater ${ }^{5}$ \\ ${ }^{1}$ Geochemistry Department, Sandia National Laboratories, Albuquerque, New Mexico, 87185- \\ 0750, USA; E-mail: rtcygan@sandia.gov \\ ${ }^{2}$ Departments of Chemistry and Geological Sciences, University College of London, and Davy \\ Faraday Research Laboratories, Royal Institution, Albermarle Street, London, W1S 4BS, UK \\ ${ }^{3}$ Johns Manville Technical Center, 10100 W. Ute Avenue, P.O. Box 625005, Littleton, Colorado \\ 80162-5005 \\ ${ }^{4}$ Department of Chemistry, Imperial College of Science, Technology, and Medicine, South \\ Kensington, London, SW7 2AY, UK \\ ${ }^{5}$ Davy Faraday Research Laboratories, Royal Institution, Albermarle Street, London, W1S 4BS, \\ UK
}

Final Version

Resubmitted to Molecular Simulation

July 2001 


\section{ABSTRACT}

We review the use of interatomic potentials to describe the bulk and surface behavior of carbonate materials. Interatomic pair potentials, describing the $\mathrm{Ca}^{2+}-\mathrm{O}$ interactions and the $\mathrm{C}-\mathrm{O}$ bonding of the $\mathrm{CO}_{3}{ }^{2-}$ anion group, are used to evaluate the lattice, elastic, dielectric, and vibrational data for calcite and aragonite. The resulting potential parameters for the carbonate group were then successfully transferred to models of the structures of rhombohedral carbonates of Mn, Fe, Mg, Ni, Zn, Co, and Cd. Simulations of the (1014) cleavage surface of calcite, magnesite, and dolomite show that these surfaces undergo relaxation leading to the rotation and distortion of the carbonate group with associated movement of cations. The influence of water on the surface structure has been investigated for monolayer coverage. The extent of carbonate group distortion is greater for the dry surfaces compared to the hydrated surfaces, and for the dry calcite relative to that for dry dolomite or magnesite. Point defect calculations for the doping of calcite indicate an increase in defect formation energy with increasing size of the substituting divalent ion. Migration energies for $\mathrm{Ca}, \mathrm{Mg}$, and $\mathrm{Mn}$ in calcite suggest a strong preference for diffusion along pathways roughly parallel to the $c$-axis rather than along the $a b$-plane.

Keywords: carbonate, calcite, shell model, surface, defect, diffusion 


\section{INTRODUCTION}

Atomistic simulations are playing an increasingly important role in the earth and materials sciences, especially in the prediction of bulk and surface structures and the physical properties of complex phases. Although most traditional examples of solid state molecular simulations involve ionic and slightly covalent solids (e.g., $\mathrm{NaCl}, \mathrm{MgO}, \mathrm{Mg}_{2} \mathrm{SiO}_{4}$, etc.) there has been relatively little interest in the simulation of the complex structures associated with systems involving metal cations and molecular-based anions (e.g., carbonate, nitrate, or sulfate phases). The three most common carbonate minerals, calcite $\left(\mathrm{CaCO}_{3}\right)$, magnesite $\left(\mathrm{MgCO}_{3}\right)$, and dolomite $\left(\mathrm{CaMg}\left(\mathrm{CO}_{3}\right)_{2}\right)$, are of special interest not just due to their occurrence in various geological environments, but also in their use in technology and manufacturing arenas [1,2]. Calcite, in particular, is used in numerous industrial applications, is a critical phase in diagenetic processes and related oil production concerns, can selectively contain various environmentally hazardous metals, and recently has been exploited in a variety of new materials relating to biomineralization and bioengineering applications.

In this paper we review the use of interatomic potentials for simulating the bulk and surface structures of calcite and related carbonate phases. The application of a recent interatomic potential model [3] to evaluate the structure and physical properties of various carbonates is highlighted, and the results are compared with experimental observation. The final section presents the results of applying the model to evaluate the defect structure and migration energy of various metal ions in the calcite structure. 


\section{CARBONATE CRYSTAL STRUCTURES}

The close-packed rhombohedral structure of the metal carbonate phases is represented in Figure 1 for the case of calcite, where a hexagonal representation is most useful in showing the alternate layering of planar carbonate groups with calcium ions. In calcite, each oxygen atom is coordinated to two calcium atoms, while in aragonite the oxygens are linked to three calcium atoms. This reflects the more close-packing nature of the aragonite structure in which the carbonate groups are rotated leading to a reduction in symmetry from hexagonal to orthorhombic. Various metal ions can be fully substituted for calcium in calcite to retain the rhombohedral structure, although only magnesite $\left(\mathrm{MgCO}_{3}\right)$, rhodochrosite $\left(\mathrm{MnCO}_{3}\right)$, siderite

$\left(\mathrm{FeCO}_{3}\right)$, and dolomite $\left(\mathrm{CaMg}\left(\mathrm{CO}_{3}\right)_{2}\right)$ typically occur in nature [4]. Various other rhombohedral metal carbonates can occur with significant solid solution in natural environments [5], but pure end-member compositions are only produced synthetically. These involve mostly transition metals to form $\mathrm{CdCO}_{3}, \mathrm{ZnCO}_{3}, \mathrm{CoCO}_{3}$, and $\mathrm{NiCO}_{3}$ phases. Metal cations having ionic radii larger than calcium usually prefer the aragonite structure where the naturally-occurring compounds, strontianite $\left(\mathrm{SrCO}_{3}\right)$ and witherite $\left(\mathrm{BaCO}_{3}\right)$ are examples.

\section{INTERATOMIC POTENTIAL MODELS}

Several theoretical treatments for the simulation of the structure and physical properties of calcite and aragonite have been published [3,6-10]. The earliest approach [10] involves a carbonate interatomic potential model based on the traditional ionic method successfully used in the treatment of simple halide and oxide solids [11,12], and then applied later for silicate phases $[13,14]$. The ionic, or nonbonded, approach involves the incorporation of spherically symmetric interactions such as electrostatic (Coulombic) and short-range (repulsion and dispersion) terms. 
Dove and co-workers [10] incorporated a three- and four-body term in their rigid ion model to account for the interaction between covalent bonds of the molecular carbonate groups $\left(\mathrm{CO}_{3}{ }^{2-}\right)$. The angle bend and torsion terms provided control of the spatial arrangement of the C-O bonds of the planar carbonate groups in place of the electrostatic repulsion of the oxygens. Empirical parameters were derived by refinement methods using the crystal structures and elastic constants for calcite and aragonite and internal vibrational frequencies of the carbonate group. A similar functional form was used by Jackson and co-workers to derive a carbonate forcefield to describe the structure of aragonite [7]. A rigid ion model, parameterized using the calcite structure and properties, was successful in predicting the orthorhombic structure of aragonite, but no details of the physical properties of either $\mathrm{CaCO}_{3}$ polymorph were reported.

A further development in potentials for the simulation of carbonate phases was provided by the inclusion of electronic polarization with a shell model [6]. The shell model incorporates a split atom representation of the oxygen ion whereby charge is partitioned between a core and a massless shell with core-shell coupling defined by a harmonic term [15]. All interaction parameters, including those for carbonate angle bend and out-of-plane displacement (torsion), were derived by empirical fitting using experimental observables for calcite, including structural data, elastic and dielectric constants, and internal and external vibrational frequencies [6,9]. Only modest improvements in the accuracy of the structural simulations were observed using the shell model compared to the results of previous rigid ion models $[6,10]$. This set of potentials was later improved by the inclusion of a Morse potential in place of a Buckingham one to better represent the covalent nature of the C-O bond in the carbonate group [16]. A quasi-harmonic approximation and lattice dynamics methods were then used to examine the role of temperature on the physical properties of calcite [16]. The simulations showed an improvement in the ability 
of the new potentials to predict cell parameters and elastic constants for calcite, but also indicated a moderate disagreement for several of the thermal expansion coefficients. The recent potential sets clearly demonstrate the need to incorporate a shell model for the oxygen atoms in the simulation of the properties of carbonate materials. Furthermore, the inclusion of a shell model in a set of interatomic potentials is critical for the simulation of surface structures, defects, and transport properties, in addition to high frequency dielectric properties. Electronic polarization contributes to energy stabilization as the electron distribution (represented by the polarization of the core and shell) of oxygens responds to changes in the local electrostatic field [17], which would be significant in the analysis of carbonate phase surfaces, defect, and migration energies.

The most recent improvement in carbonate potentials is that of Fisler and co-workers [3] in which a general set of forcefield parameters were derived that could be easily transferred to a wide range of metal carbonate phases. As with previous models the Fisler and co-workers model (FGC) [3] incorporates an electrostatic term with a Buckingham potential to describe the energy of the ion-ion interactions:

$$
E_{i j}=\frac{q_{i} q_{j}}{r_{i j}}+A_{i j} \exp \left(-r_{i j} / \rho_{i j}\right)-\frac{C_{i j}}{r_{i j}^{6}}
$$

$E_{i j}$ represents the interaction energy for two ions of charge $q_{i}$ and $q_{j}$ (unit electron charge) separated by a distance $r_{i j}$. The exponential term is associated with the repulsion of the interacting ions at very short interatomic distances, and the last term is for the attractive shortrange van der Waals interactions. The Buckingham potential parameters $A_{i j}, \rho_{i j}$, and $C_{i j}$ are empirically derived by fitting of the model potential to the observed structural and physical property data. The polarization energy for oxygen is represented by: 


$$
E^{\text {polar }}=1 / 2 k d^{2}
$$

where $k$ is a harmonic force constant and $d$ is the distance between the shell and core of the oxygen. During the energy optimization calculation of a carbonate structure, the shell positions are allowed to relax about the oxygen core resulting in a local dipole moment that simulates the electronic polarization. The combination of the dipolar polarization at each oxygen in turn controls the quadrupole moment of the carbonate anion.

The primary improvement of the FGC potential model over previous models is the introduction of separate Buckingham potentials to describe intramolecular and intermolecular OO interactions. The authors recognized the vastly different behavior of O-O interactions within the carbonate group in comparison to those associated with carbonate-carbonate interactions. A three-body O-C-O term and a torsional O-C-O-O were included to help constrain the internal geometry of the carbonate group, as done previously. The shell model for oxygen was further refined by the inclusion of a fourth order term. This helped to improve the accuracy of the predicted vibrational frequencies of calcite and to properly model the relative stability of aragonite with respect to calcite. Finally, a Morse potential was used to describe the covalent bonding of the C-O interaction in the carbonate group. Coulombic interactions are excluded within the molecular carbonate group.

The lattice energy of an ionic crystal is typically evaluated by the summation of Equation (1) over all possible atomic interactions in a three-dimensional structure. An Ewald summation procedure is used whereby the electrostatics are partially summed in reciprocal space to ensure proper convergence of the long-range interactions [18]. This procedure is slightly modified for the more complex structures of the carbonate phases where the covalent carbonate group requires special attention. Energy contributions for the Morse, angle bend, torsion, and 
polarization terms of the carbonate groups are also included in the evaluation. The computer program GULP [19] was used to first derive the potential parameters for the FGC shell model and then to perform the energy minimizations required for the bulk structure simulations of the carbonate phases. The parameterization process involved the relaxed fitting [20] of parameters to the structures and elastic constants of calcite and aragonite, the dielectric properties of calcite, and the vibrational frequencies for the internal modes of calcite and aragonite (zero wavevector). The relaxed fitting method involves using fully-relaxed structures throughout the fitting procedure. Additionally, Fisler and co-workers [3] used the resulting model parameters as a basis for deriving interaction parameters for several divalent metal cations associated with other rhombohedral carbonate phases. They included recent crystallographic and compressibility data for magnesite and transition metal carbonates $[4,21,22]$ to derive metal-oxygen Buckingham parameters suitable for simulating these carbonate phases.

\section{BULK STRUCTURES AND PROPERTIES}

Table I provides a comparison of the experimental and calculated structures, as well as bulk moduli for calcite, aragonite, and several rhombohedral metal carbonates. The predicted values were derived using the FGC shell model and were taken from Fisler and co-workers [3]. Lattice parameters for the end-member carbonate phases are all in agreement with the observed values by a margin of less than one percent. The $c$-axis parameter for the mixed-metal carbonate

dolomite is underestimated by approximately two percent. Lattice energies for the rhombohedral phases generally increase with increasing size of the cation radius. Bulk moduli derived from the predicted elastic constants (see discussion by Nye [23]) are in very good agreement with the experimental data [22], although the FGC parameterization used the observed elastic data in the 
fitting procedure. However, it should be noted that only two parameters were fitted in the case of each structure. A series of energy minimizations for both calcite and aragonite as a function of increasing pressure predicts the phase transition from calcite to aragonite at approximately 2.4 kbar. This value is consistent with the extrapolated results of experimental observations of the transition $[24,25]$. It is important to note that predicted transition pressure is quite sensitive to the electronic polarization of the oxygens; the carbonate groups must rotate through an energy barrier associated with the deformation of the oxygen valence cloud that occurs during the calcite-aragonite transition.

A comparison of the experimental and calculated physical properties of calcite and aragonite is presented in Table II. As noted for several table entries, not all of the elastic and dielectric constants were used in the parameterization scheme for the FGC shell model. Yet, the model provides reasonable predictions for the calcite and aragonite properties. While all elastic constants for calcite are accurately reproduced, only the diagonal components of the elastic tensor for aragonite are predicted within reasonable accuracy. Internal vibration modes for the carbonate group for both structures are accurately reproduced by the shell model. Low frequency vibrations associated with Ca-O modes are incorporated in the FGC model by fitting the elastic properties rather than the vibrational frequencies. In general, the favorable comparison of structures and properties presented in Tables I and II demonstrates the success of the FGC shell model in providing a significant improvement in the prediction of structure and second derivative properties over previous carbonate models.

\section{SURFACE STRUCTURES}


A useful application for the carbonate forcefield approach is in the simulation of the carbonate-solution interface. Fluid-mineral interactions control numerous environmental and geochemical processes, especially those related to carbonate mineral growth, dissolution, and sorption processes [26-28]. Therefore, it is important to use an accurate atomistic description in order to assess the atomic arrangement of the surface layers and their interaction with solute and solvent species. This is especially true when more computationally-expensive quantummechanical methods are currently unable to deal with the large number of atoms needed to represent the more complex carbonate surfaces and interfaces.

Several studies have examined the equilibrium surfaces and morphology of calcite. Titiloye and co-workers [29] evaluated the low index surfaces of calcite and were able to derive Wulff-plot morphologies that are in general agreement with experimental observation. An accurate carbonate potential can be used to evaluate the surface and attachment energies for various crystal cleavage surfaces and deduce the morphology of the crystal [29-31]. The influence of ionic additives such as $\mathrm{Li}^{+}, \mathrm{Mg}^{2+}$, and $\mathrm{HPO}_{4}{ }^{2-}$ on calcite morphology has also been simulated [29]. The modeling approach was later extended for the simulation of dolomite surfaces [32].

More recently, de Leeuw and co-workers [30,31,33-35] have completed a series of simulation studies of calcite surfaces based on the carbonate potential of Pavese and co-workers [16]. Noteworthy in this work is the incorporation of an adsorbed water layer onto the calcite surface model $[30,31]$ leading to further stabilization of the calcite surfaces. The dominant (1014) cleavage surface of calcite remains the most stable surface in both dry and wet environments. Additionally, the sorption of $\mathrm{Mg}^{2+}$ and $\mathrm{Cd}^{2+}$ onto calcite surfaces was examined with the simulation results indicating the preference of cadmium for incorporation into the bulk 
calcite crystal while magnesium is maintained on the surface [33]. Molecular dynamics

simulations of the (1014) calcite surface suggest the formation of kink sites on the obtuse step of dissolving edge controls calcite dissolution [34]. These studies demonstrate the importance of molecular simulations in providing a theoretical description of complex surface processes for calcite, and how the theoretical models assist the experimentalist in evaluating competing models to understand mechanisms and explain experimental observations.

The atomistic simulation of a carbonate surface first requires the creation of the surface by cleaving a bulk crystal, and then the relaxation of the surface atoms to obtain an energy minimized surface structure [36-38]. For carbonate materials, and in particular calcite, there are numerous surfaces to evaluate in order to derive the equilibrium surface morphology. Typically, the low Miller index surfaces are most commonly observed and dominate the morphology [29]. Surface energies are derived by representing the periodic surface structure as two regions: an upper section where the near-surface atoms are allowed to freely translate and relax, and a lower section where the atoms are fixed at positions associated with the bulk equilibrium structure. The specific surface energy $\gamma$ for a given crystal plane is given as:

$$
\gamma=\frac{E_{\mathrm{s}}-E_{\mathrm{b}}}{\mathrm{A}}
$$

where $E_{\mathrm{s}}$ is the lattice energy summed over the surface atoms and $E_{b}$ is the bulk lattice energy for the corresponding number of atoms. The most stable surfaces are identified by the lowest surface energies and an equilibrium morphology can be predicted by the construction of a Wulff plot [39]. Often dipolar surfaces, such as the (0001) surface of calcite having alternating layers of calcium ions and carbonate groups, occur and require reconstruction or the addition of 
charged species in order to stabilize the model surface. Interestingly, the two original sets of carbonate potentials [6,7] used in the above studies provided equivalent morphologies for calcite.

Recently, the FGC shell model was used to describe and compare the structure of calcite, magnesite, and dolomite surfaces [40,41]. Calculations were performed using the MARVIN code [38] with the FGC potentials to generate energy-minimized structures for the dominant (1014) surfaces. MARVIN uses two-dimensional periodic boundary conditions to evaluate the surface energy, and can be used to obtain fully-relaxed surface structures. Figure 2 presents the results of the calculations for the vacuum-terminated surfaces. The optimized calcite surface exhibits significant rotation and distortion of the carbonate groups accompanied by a displacement of the calcium ions away from the surface. The second layer shows similar carbonate group distortion but the calcium ions exhibit less displacement. Interestingly, the surface structures generated with the FGC model exhibit carbonate groups that form layers with alternating convex and concave distortions with respect to the surface plane that are less severe for subsequent layers moving away from the surface-vacuum interface. The magnesite (10ī) surface has less carbonate distortion and magnesium ion displacement than observed for the calcite model. Relaxation of the magnesite surface is less pronounced with much of the displacement occurring within the first four surface layers. The surface for the mixed metal structure of dolomite exhibits both carbonate group distortion and metal ion displacement, with the magnesium being more displaced in the topmost surface layer than calcium, but less in subsequent layers. The carbonate groups exhibit less rotation with respect to the bulk position in dolomite than those calculated for calcite.

Hydrated models of the (1014) carbonate surfaces were examined by positioning a monolayer of water molecules above the surface (one water per cation), and then allowing the 
carbonate surface and water to relax to their minimum energy configuration. A polarizable water potential [42] including a shell on the oxygen was used in the calculations. The simulations indicate that for each of the carbonate surfaces the relaxation from the initial configuration is limited to the first three surface layers, and the extent of the relaxation is significantly reduced compared to those for the dry surfaces. For example, first-layer calcium ion in calcite is shifted from its bulk position by only $0.26 \AA$ when hydrated compared to $1.05 \AA$ for the dry surface. Additionally, there is a reduction in the distortion associated with the torsional angle of the carbonate groups as a function of surface layer (depth). Figure 3 provides a comparison of this distortion trend for calcite under dry and wet conditions. The out-of-plane distortion is controlled by the torsion energy term as represented by the dihedral angle $\varphi$ for the O-C-O-O configuration of the carbonate group.

Water molecules are associated with each of the exposed metal sites in order to complete the octahedral coordination of the metal ion with oxygens. The water molecules lie almost flat on the calcite surface and have a mean $\mathrm{Ca}-\mathrm{O}_{\text {water }}$ distance of $2.55 \AA$ that is in excellent agreement with experimental X-ray reflectivity result of $2.5 \AA$ [43]. There is some variation of the disposition of the waters on the dolomite (1014) surface compared to that for either the calcite and magnesite surfaces. The alternating nature of magnesium and calcium cations results in a crenellation for the first surface layer of the dolomite. The coordination shell of magnesium being significantly smaller than that for calcium (ionic radii: $\mathrm{Mg}^{2+}=0.66 \AA$ versus $\mathrm{Ca}^{2+}=0.99$ Å) $[44,45]$ contributes to significant out-of-plane distortions of the carbonated groups coupled with displacement of the magnesium ions into the surface. This behavior leads to a canting of the surface water molecules such that the magnesium-associated waters are oriented with their dipole pointing away from the surface. As a result, the strongest hydrogen bonds occur with 
dolomite rather than calcite or magnesite. The end-member carbonates display a surface structure where the water molecules form a more regularly-distributed monolayer.

Overall, as noted previously [30,31] for calcite, water helps to stabilize the (1014) surface of the carbonate mineral surface. In the dry case, the FGC shell model provides surface energies based on Equation (3) of 0.322, 0.247 , and $0.256 \mathrm{~J} / \mathrm{m}^{2}$ for calcite, dolomite, and magnesite, respectively; when fully hydrated with a monolayer of water, the energies decrease respectively to $0.232,0.165$, and $0.084 \mathrm{~J} / \mathrm{m}^{2}[40,41]$. The dolomite (1014) surface is the most stable of the three examples when dry, while the magnesite (1014) surface is the most stable when wet. The calcite surface is the least stable when either dry or wet.

\section{DEFECTS AND MIGRATION ENERGIES}

The defect structure and cation diffusion properties of calcite and other metal carbonate phases is of critical importance in understanding numerous transport-controlled processes including creep, chemical alteration, and dissolution. Chemical zoning and diffusional homogenization of carbonate phases are of also of significant interest due to the persistence of complex zoning patterns recently found in the carbonate phases of the Allan Hills Martian meteorite [46]. The ability to extract useful thermal histories from the observed zoning patterns of iron, magnesium, calcium, and manganese in the carbonate phases of the meteorite inclusions will rely on our understanding of the mechanisms of diffusion $[47,48]$.

Diffusion processes in solids are directly linked to the defect structure and migration pathways with the most dominant transport being controlled by the energetics. Intrinsic diffusion processes are controlled by the thermal generation of defects in the solid plus the migration energy needed to overcome the energy barrier found between two diffusion sites. In 
contrast, an extrinsic diffusion process has an activation energy that is equal to the migration energy since the defect structure is defined by the composition, especially with the presence of multivalent impurities [49]. In principal, the defect formation and cation migration energies for a solid phase can be evaluated within the constraints of the Born model and a suitable set of interatomic potentials. The occurrence of the highly covalent carbonate anion in the calcite is not problematic in this effort since the material can be treated as a highly ionic solid comprised of metal cations and the $\mathrm{CO}_{3}{ }^{2-}$ molecular anions. Again, as with surfaces and some physical properties, it is important to incorporate electronic polarization with the shell model in order to accurately describe the energies [50,51].

\section{Defects}

Defect calculations for calcite were performed by Fisler and co-workers [3] as part of their development of the potentials for the FGC shell model. A Mott-Littleton approach [52] as implemented in GULP [19] was used to evaluate the energy associated with atomic displacements about a defect site. Convergence of the lattice energy for such a defect calculation is obtained by treating the system as two parts: an inner region of several hundred atoms in which all are allowed to explicitly relax in response to the defect site, plus a hybrid outer region where either a harmonic response occurs or an implicit polarization of sublattices is used. This computational approach is approximate, yet generally assures the proper convergence of the defect energy. The total defect formation energy can then be calculated from the difference between the energies of the defective lattice and the perfect bulk lattice.

Table III provides the results of the defect calculations for calcite obtained by this method. Values represent the defect energy after convergence for adequately-sized regions 
(usually for inner regions of 10 to $12 \AA$ and an outer region of $20 \AA$ ). The calcium vacancy and carbonate vacancy defect energies represent the energy for the formation of a charged and isolated vacancy with the ion being removed to an infinite distance from the crystal. The reported values are quite large and have not been corrected for removing the ion to the crystal surface. Therefore, the energies represent a maximum. A more helpful defect energy is that for the Schottky defect pair that involves both a calcium vacancy and a carbonate vacancy, which for this study are located along the $c$-axis of the hexagonal cell and separated by a distance of 4.46 A. In comparison to the isolated vacancies, the Schottky defect is stabilized by the local distortions of the lattice about the defect and electrostatic interaction of the charged defect centers. Table III also includes the formation energies for substitutional defects where various metal cations replace the calcium in calcite. The negative energies reflect the stabilizing influence of forming compositional defects involving divalent metals having smaller ionic sizes than calcium ( $0.99 \AA)$. Of course, the exact values of the defect energies involving metal substitutions will depend on the observables (structure, bulk moduli, etc.) used in the parameterization of the interatomic potentials [3].

\section{Cation Migration}

Migration energies are calculated by determining the transition state that occurs as an energy barrier between a cation and a defect site. The computer program GULP [19] was used to examine the migration energies for calcium, magnesium, and manganese cations in calcite along two different pathways, one perpendicular to the $c$-axis, in the $a b$-plane, and one roughly parallel to the $c$-axis based on the hexagonal representation of the unit cell. Either diffusion mechanism would occur in calcite assuming the preexistence of the cation vacancies. We are 
assuming that diffusion is controlled by a vacancy mechanism, rather than an interstitial process, which for close-packed structures is usually the case [49]. A schematic of the two diffusion paths is presented in Figure 4 for the hexagonal representation of calcite. The transition state search approach used in the calculations incorporates a mode-following algorithm to identify the true transition state and determine the negative eigenvalue associated with the correct Hessian matrix. The corresponding eigenvector will be in the direction of the diffusion coordinate at the saddle point.

Table IV presents the results of the migration energy calculations. Migration energies are lowest for diffusion roughly parallel to the $c$-axis for each of the three cations. The energetically-favored route involves a zigzag path in which the metal cation diffuses across a carbonate layer to the next cation layer. The cation-cation distance along this path is $4.10 \AA$. Oxygen atoms of the coordinating carbonate groups polarize as the cation passes between layers. Specifically, the sub-parallel direction corresponds to a diffusion pathway that can be either along the approximate [1014] direction or exactly parallel to [0001] depending on the choice of successive cation jump steps. Migration energies for diffusion within the $a b$-plane are almost twice as high when compared to those for the parallel $c$-axis path. Here, cation diffusion is restricted to the plane between the carbonate layers with at least two oxygens from each neighboring carbonate group effecting the cation movement. The cation jump distance for this case is $4.99 \AA$.

The migration energies for calcium for either pathway, in the case of calcite, are largest of those for the three metal cations examined. Migration energy values for magnesium are the lowest of the three, with manganese values being similar to, but less than, those for calcium. Not surprisingly, this trend follows the behavior expected for cation diffusion based on an ionic radii 
arguments for divalent metals $\left(\mathrm{Ca}^{2+}=0.99 \AA, \mathrm{Mn}^{2+}=0.80 \AA\right.$, and $\mathrm{Mg}^{2+}=0.65 \AA$ )—the smaller the cation, the less energy required to migrate along a given pathway. Additionally, one would expect electronic polarization for these cations to have some influence, with $\mathrm{Ca}^{2+}$ being the most polarizable of the three [53], but the FGC shell model includes only polarization for oxygens.

Recent cation diffusion measurements for calcite suggest activation energies to be approximately $0.80 \mathrm{eV}$ for magnesium diffusion and $2.2 \mathrm{eV}$ for calcium diffusion in calcite [47]. Another experimental study observed activation energies for magnesium and calcium diffusion in calcite to be similar at approximately $2.8 \mathrm{eV}$ [54]. Assuming an extrinsic diffusion process where the defect concentrations are controlled by impurities, the activation energy should be equivalent to the migration energy [51]. The $0.80 \mathrm{eV}$ experimental activation energy for magnesium falls close to the predicted $1.00 \mathrm{eV}$ value based on the FGC shell model. However, the other experimental magnesium value and the experimental activation energies for calcium are quite high compared to the model values for the $c$-axis pathway. This difference can be reconciled in either of two ways. First, the experimental results represent an intrinsic diffusion process where the defect formation energy is a component of the observed activation energy. Our previous defect calculations suggest that the estimated value for the formation of a $\mathrm{Ca}^{2+}$ $\mathrm{CO}_{3}{ }^{2-}$ Schottky pair defect is fairly large (see above), but other defect mechanism possibilities still need to be addressed. Secondly, the $2.2 \mathrm{eV}$ and $2.8 \mathrm{eV}$ values for the experimental activation energy are similar to the migration energy for calcium perpendicular to the $c$-axis (2.5 $\mathrm{eV}$ ). Noteworthy, is that the experimental studies typically determine diffusion coefficients for a single direction, usually perpendicular to common cleavage surfaces such as the (1014) and (1011) planes, but not along either the [1010] or [0110] directions (that is, within the $a b$-plane). Farver and Yund [55] suggested that calcite does not exhibit significant anisotropy for calcium 
diffusion, yet our calculations suggest otherwise, at least in terms of migration energies. In part, this would help explain the differences between observed and predicted activation energiesother low energy diffusion mechanisms may be contributing to the observed cation diffusion in calcite.

\section{CONCLUSIONS}

The FGC shell model provides a general method for successfully describing the ionic and molecular interactions associated with metal carbonate solids. A classical atomistic approach is successful in predicting the bulk structure and physical properties of calcite, aragonite, and various rhombohedral divalent metal carbonate phases. Application of the carbonate potential model to surface structures and surface energy calculations, and in defect formation and migration energy analysis provides a sound theoretical basis for evaluating numerous geochemical and materials processes. Although recent quantum mechanical studies $[8,56,57]$ may provide a more accurate description of the electronic structure of carbonate solids, the computational cost of Hartree-Fock or density functional methods currently prohibit their routine use in modeling complex bulk or surface configurations involving many hundreds of atoms where many states may have to be examined. 


\section{ACKNOWLEDGMENTS}

The senior author is appreciative of funding provided by the U.S. Department of Energy, Office of Basic Energy Sciences, Geosciences Research Program. Kate Wright and Julian Gale would both like to thank the Royal Society for support through the University Research Fellowship program. Sandia is a multiprogram laboratory operated by Sandia Corporation, a Lockheed Martin company, for the United States Department of Energy under contract DEAC04-94AL85000. 


\section{REFERENCES}

[1] D. Donnert and M. Salecker, "Elimination of phosphorus from municipal and industrial waste water," Water Sci. Technol. 40 (4-5), 195-202 (1999).

[2] A. Richter, D. Petzold, H. Hofman et al., "Production, properties and application of calcium carbonate powders: 1. Production of calcium carbonate by precipitation from solutions," Chemische Technik 47 (6), 306-313 (1995).

[3] D. K. Fisler, J. D. Gale, and R. T. Cygan, "A shell model for the simulation of rhombohedral carbonate minerals and their point defects," Am. Mineral. 85, 217-224 (2000).

[4] H. Effenberger, K. Mereiter, and J. Zemann, "Crystal structure refinements of magnesite, calcite, rhodochrosite, siderite, smithsonite, and dolomite, with discussion of some aspects of the stereochemistry of calcite-type carbonates," Z. Kristallogr. 156 (3-4), 233-243 (1981).

[5] V. Bermanec, G. Sijaric, G. Kniewald et al., "Gaspeite and associated Ni-rich minerals from veins in altered ultrabasic rocks from Dubostica, Bosnia and Herzegovina," Can. Mineral. 38 (pt.6), 1371-1376 (2000).

[6] A. Pavese, M. Catti, G. D. Price et al., "Interatomic potentials for $\mathrm{CaCO}_{3}$ polymorphs (calcite and aragonite), fitted to elastic and vibrational data," Phys. Chem. Miner. 19, 80-87 (1992).

[7] R. A. Jackson, P. A. Meenan, G. D. Price et al., "Deriving empirical potentials for molecular ionic materials," Mineral. Mag. 59, 617-622 (1995).

[8] M. Catti and A. Pavese, "Quantum-mechanical and classical simulations of Mg-Ca carbonates," in Modelling of Minerals and Silicated Materials, edited by B. Silvi and P. D'Arco (Kluwer Academic, Dordrecht, 1997), pp. 113-156.

[9] M. Catti, A. Pavese, and G. D. Price, "Thermodynamic properties of $\mathrm{CaCO}_{3}$ calcite and aragonite: A quasi-harmonic calculation," Phys. Chem. Miner. 19, 472-479 (1993).

[10] M. T. Dove, B. Winkler, M. Leslie et al., "A new interatomic potential model for calcite: Applications to lattice dynamics studies, phase transition, and isotopic fractionation.," Am. Mineral. 77, 244-250 (1992).

[11] W. R. Busing, "An interpretation of the structures of alkaline earth chlorides in terms on interionic forces," Trans. Am. Cryst. Assoc. 6, 57-72 (1970).

[12] C. R. A. Catlow, W. C. Mackrodt, and V. R. Saunders, "Computer modeling of complex and massively disordered solids," in Computer Simulation in the Physics and Chemistry of Solids (Science Research Council, Daresbury Laboratory, 1980), pp. 36-44.

[13] C. R. A. Catlow, "Computer modeling of silicates," in Physical Properties and Thermodynamic Behaviour of Minerals, edited by E. K. H. Salje (D. Reidel, 1988), pp. 619-638. 
[14] C. R. A. Catlow, J. M. Thomas, S. C. Parker et al., "Simulating silicate structures and the structural chemistry of pyroxenoids," Nature 295 (5851), 658-662 (1982).

[15] B.G. Dick and A.W. Overhauser, "Theory of the dielectric constants of alkali halide crystals," Phys. Rev. 112, 90-103 (1958).

[16] A. Pavese, M. Catti, S. C. Parker et al., "Modelling of the thermal dependence of structural and elastic properties of calcite, $\mathrm{CaCO}_{3}$," Phys. Chem. Miner. 23 (2), 89-93 (1996).

[17] A. C. Lasaga, "Defect calculations in silicates: Olivine," Am. Mineral. 65, 1237-1248 (1980).

[18] M. P. Tosi, "Cohesion of ionic solids in the Born model," Solid State Phys. 131, 533-545 (1964).

[19] J. D. Gale, "GULP: A computer program for the symmetry-adapted simulation of solids," J. Chem. Soc., Faraday Trans. 93, 629-637 (1997).

[20] J. D. Gale, "Empirical potential derivation for ionic materials," Philos. Mag. B 73, 3-19 (1996).

[21] D. L. Graf, "Crystallographic tables for the rhombohedral carbonates," Am. Mineral. 46, 629-637 (1961).

[22] J. Zhang and R. J. Reeder, "Comparative compressibilities of calcite-structure carbonates: Derivations from empirical relations," Am. Mineral. 84, 861-870 (1999).

[23] J. F. Nye, Physical properties of crystals (Oxford University Press, Oxford, 1985).

[24] W. A. Crawford and A. L. Hoersch, "Calcite-aragonite equilibrium from $50^{\circ}$ to $150^{\circ} \mathrm{C}$," Am. Mineral. 57, 995-998 (1972).

[25] A. L. Boettcher and P. J. Wyllie, "Revision of the calcite-aragonite transition," Nature (London) 213, 792-793 (1967).

[26] S. L. S. Stipp, "Toward a conceptual model of the calcite surface: Hydration, hydrolysis, and surface potential," Geochim. Cosmochim. Acta 63 (19-20), 3121-3131 (1999).

[27] L. Cheng, N. C. Sturchio, and M. J. Bedzyk, "Local structure of $\mathrm{Co}^{2+}$ incorporated at the calcite surface: An x-ray standing wave SEXAFS study," Phys. Rev. B: Condens. Matter 61 (7), 4877-4883 (2000).

[28] P. van Cappellen, L. Charlet, W. Stumm et al., "A surface complexation model of the carbonate mineral-aqueous solution interface," Geochim. Cosmochim. Acta 57 (15), 3505-3518 (1993).

[29] J. O. Titiloye, S. C. Parker, and S. Mann, "Atomistic simulation of calcite surfaces and the influence of growth additives on their morphology," J. Cryst. Growth 131, 533-545 (1993). 
[30] N. H. de Leeuw and S. C. Parker, "Atomistic simulation of the effect of molecular adsorption of water on the surface structure and energies of calcite surfaces," J. Chem. Soc., Faraday Trans. 93, 467-475 (1997).

[31] N. H. de Leeuw and S. C. Parker, "Surface structure and morphology of calcium carbonate polymorphs calcite, aragonite, and vaterite: An atomistic approach," J. Phys. Chem. B 102 (16), 2914-2922 (1998).

[32] J. O. Titiloye, N. H. de Leeuw, and S. C. Parker, "Atomistic simulation of the differences between calcite and dolomite surfaces," Geochim. Cosmochim. Acta 62 (15), 2637-2641 (1998).

[33] N. H. de Leeuw and S. C. Parker, "Modeling absorption and segregation of magnesium and cadmium ions to calcite surfaces: Introducing MgCO3 and CdCO3 potential models," J. Chem. Phys. 1112 (9), 4326-4333 (2000).

[34] N. H. de Leeuw, S. C. Parker, and J. H. Harding, "Molecular dynamics simulation of crystal dissolution from calcite steps," Phys. Rev. B: Condens. Matter 60, 13792-13799 (1999).

[35] N. H. de Leeuw, G. W. Watson, and S. C. Parker, "Atomistic simulation of adsorption of water on three-, four- and five-coordinated surface sites of magnesium oxide," J. Chem. Soc., Faraday Trans. 92, 2081-2091 (1996).

[36] P. W. Tasker, "The surface energies, surface tensions and surface structure of the alkali halide crystals," Philos. Mag. A 39, 119-136 (1979).

[37] P. W. Tasker and A. M. Stoneham, "Computer simulation of ceramic surfaces and interfaces," Conference on Ceramic Surfaces and Surface Treatments, (1984).

[38] D. H. Gay and A. L. Rohl, "MARVIN: A new computer code for studying surfaces and interfaces and its application to calculating the crystal morphologies of corundum and zircon," J. Chem. Soc., Faraday Trans. 91, 925-936 (1995).

[39] W. T. Lee, E. K. H. Salje, and M. T. Dove, "Effect of surface relaxations on the equilibrium growth morphology of crystals: Platelet formation," J. Phys.: Condens. Matter 11 (38), 73857410 (1999).

[40] K. Wright, R. T. Cygan, and B. Slater, "Atomistic simulations of the (1014) surface of carbonate minerals," in Morphololgy and Dynamics of Crystal Surfaces in Complex Molecular Systems, edited by J. de Yoreo, W. Casey, A. Malkin et al. (Materials Research Society, Pittsburgh, 2000), Vol. 620, pp. M2.2.1-M2.2.6.

[41] K. Wright, R. T. Cygan, and B. Slater, "Structure of the (1014) surfaces of calcite, dolomite, and magnesite under wet and dry conditions," Phys. Chem. Chem. Phys. 3, 839-844 (2001).

[42] N. H. de Leeuw and S. C. Parker, "Molecular-dynamics simulation of MgO surfaces in liquid water using a shell-model potential for water," Phys. Rev. B: Condens. Matter 58, 1390113908 (1998). 
[43] P. Fenter, P. Geissbuhler, E. DiMasi et al., "Surface speciation of calcite observed in situ by high-resolution X-ray reflectivity," Geochim. Cosmochim. Acta 64 (7), 1221-1228 (2000).

[44] R. D. Shannon and C. T. Prewitt, "Revised values of effective ionic radii," Acta Crystallogr., Sect. B: Struct. Sci. 26, 1046-1048 (1970).

[45] R. D. Shannon and C. T. Prewitt, "Effective ionic radii in oxides and fluorides," Acta Crystallogr., Sect. B: Struct. Sci. 25, 925-946 (1969).

[46] R. P. Harvey and H. Y. McSween, "A possible high-temperature origin for the carbonates in the Martian meteorite ALH84001," Nature 382 (6586), 49-51 (1996).

[47] A. J. R. Kent, I. D. Hutcheon, F. J. Ryerson et al., "The temperature of formation of carbonate in Martian meteorite ALH84001: Constraints from cation diffusion," Geochim. Cosmochim. Acta 65 (2), 311-321 (2001).

[48] D. K. Fisler and R. T. Cygan, "Cation diffusion in carbonate minerals: Determining closure temperatures and the thermal history for the ALH84001 meteorite," Meteoritics Planet. Sci. 33, 785-789 (1998).

[49] L. W. Barr and A. B. Lidiard, "Defects in ionic crystals," Physical Chemistry; An Advance Treatise, Jost. Edition 10, 151-228 (1971).

[50] C. R. A. Catlow, I. D. Faux, and M. J. Norgett, "Shell and breathing shell model calculations for defect formation energies and volumes in magnesium oxide," J. Phys. C: Solid State Phys. 9, 419-429 (1976).

[51] A. C. Lasaga, "The atomistic basis of kinetics: Defects in minerals," in Kinetics of Geochemical Processes (1981), Vol. 8, pp. 1-50.

[52] N. F. Mott and M. J. Littleton, "Conduction in polar crystals. I. Electrolytic conduction in solid salts.," Transactions of the Faraday Society 34, 485-499 (1938).

[53] H. W. Jaffe, Crystal Chemistry and Refractivity (Cambridge University Press, Cambridge, 1988).

[54] D. K. Fisler and R. T. Cygan, "Diffusion of Ca and Mg in calcite," Am. Mineral. 84, 13921399 (1999).

[55] J. R. Farver and R. A. Yund, "Volume and grain boundary diffusion of calcium in natural and hot-pressed calcite aggregates," Contrib. Mineral. Petrol. 123, 77-91 (1996).

[56] M. Catti, A. Pavese, R. Dovesi et al., "Static lattice and electron properties of $\mathrm{MgCO}_{3}$ (magnesite) calculated by ab initio periodic Hartree-Fock methods," Phys. Rev. B: Condens. Matter 47 (15), 9189-9198 (1993).

[57] H. Ruuska, P. Hirva, and T. A. Pakkanen, "Cluster models for calcite surfaces: Ab initio quantum chemical studies," J. Phys. Chem. B 32, 6734-6740 (1999). 
[58] H. Y. Zhang and M. S. T. Bukowinski, "Modified potential-induced breathing model of potentials between closed-shell ions," Phys. Rev. B: Condens. Matter 44 (6), 2495-2503 (1991).

[59] A. dal Negro and L. Ungaretti, "Refinement of the crystal structure of aragonite," Am. Mineral. 56, 768-773 (1971).

[60] V. L. Borodin, V. I. Lyntin, V. V. Ilyukhin et al., "Isomorphic octavite-calcite series," Soviet Physic Doklady 24, 226-227 (1979).

[61] N. L. Ross and R. J. Reeder, "High-pressure structural study of dolomite and ankerite," Am. Mineral. 82, 412-421 (1992).

[62] D. P. Dandekar and A. L. Ruoff, "Temperature dependence of the elastic constants of calcite between 160 and 300 K," J. Appl. Phys. 39, 6004-6009 (1968).

[63] R. F. S. Hearmon, "The elastic constants of anisotropic minerals," Rev. Mod. Phys. 18, 409440 (1946).

[64] W. A. Deer, R. A. Howie, and J. Zussman, An Introduction to the Rock Forming Minerals (Longmans, London, 1966).

[65] G. W. C. Kay and T. H. Laby, Table of Physical and Chemical Constants (Longmans, London, 1982).

[66] W. B. White, "The carbonate minerals," in Infrared Spectroscopy of Minerals, edited by V. C. Farmer (Mineralogical Society, London, 1974), pp. 227- 284. 
Table I. Comparison of metal carbonate structural properties and bulk moduli

\begin{tabular}{|c|c|c|c|c|c|c|c|c|c|c|}
\hline \multirow[t]{2}{*}{ Composition } & \multirow{2}{*}{$\begin{array}{c}\text { Calculated Lattice } \\
\text { Energy (eV) }\end{array}$} & \multirow[t]{2}{*}{ a $(\AA)$} & \multirow[t]{2}{*}{$\Delta \mathrm{a}(\%)$} & \multirow[t]{2}{*}{ b $(\AA)$} & \multirow[t]{2}{*}{$\Delta \mathrm{b}(\%)$} & \multirow[t]{2}{*}{ c $(\AA)$} & \multirow[t]{2}{*}{$\Delta \mathrm{c}(\%)$} & \multicolumn{3}{|c|}{ Bulk Modulus } \\
\hline & & & & & & & & $\begin{array}{c}\text { Calculated } \\
\text { (GPa) }\end{array}$ & $\begin{array}{c}\text { Experimental } \\
\text { (GPa) }\end{array}$ & $\Delta(\%)$ \\
\hline $\mathrm{CaCO}_{3}$ (calcite) ${ }^{3}$ & -85.31 & 4.99 & 0.01 & 4.99 & & 17.06 & -0.01 & 77 & $73^{7}$ & 5.5 \\
\hline $\mathrm{CaCO}_{3}(\mathrm{arag})^{2}$ & -85.30 & 5.75 & 0.31 & 4.94 & 0.48 & 7.92 & 0.61 & 71 & $48^{8}$ & 48 \\
\hline $\mathrm{CdCO}_{3}{ }^{4}$ & -87.76 & 4.90 & -0.51 & 4.90 & & 16.39 & -0.63 & 98 & $100^{1}$ & -2.0 \\
\hline $\mathrm{MnCO}_{3}{ }^{3}$ & -89.55 & 4.78 & 0.29 & 4.78 & & 15.56 & -0.51 & 108 & $108^{1}$ & 0.0 \\
\hline $\mathrm{FeCO}_{3}{ }^{3}$ & -90.79 & 4.73 & 0.80 & 4.73 & & 15.25 & -0.87 & 121 & $117^{1}$ & 3.4 \\
\hline $\mathrm{ZnCO}_{3}{ }^{3}$ & -91.30 & 4.67 & 0.45 & 4.67 & & 14.88 & -0.94 & 123 & $123^{1}$ & 0.0 \\
\hline $\mathrm{CoCO}_{3}{ }^{5}$ & -91.37 & 4.67 & 0.25 & 4.67 & & 14.89 & -0.51 & 124 & $124^{1}$ & 0.0 \\
\hline $\mathrm{NiCO}_{3}{ }^{5}$ & -92.59 & 4.64 & 0.65 & 4.64 & & 14.73 & -0.03 & 138 & $131^{1}$ & 5.3 \\
\hline $\mathrm{MgCO}_{3}{ }^{3}$ & -91.16 & 4.68 & -0.96 & 4.68 & & 14.92 & 0.69 & 122 & $107^{1}$ & 14.0 \\
\hline $\mathrm{MgCa}\left(\mathrm{CO}_{3}\right)_{2}{ }^{6}$ & -88.32 & 4.85 & 0.89 & 4.85 & & 15.82 & -1.16 & 94 & $91^{1}$ & 3.3 \\
\hline
\end{tabular}

${ }^{1}$ Experimental bulk moduli [58]

${ }^{2}$ Experimental lattice parameters [59]

${ }^{3}$ Experimental lattice parameters [4]

${ }^{4}$ Experimental lattice parameters [60]

${ }^{5}$ Experimental lattice parameters [21]

${ }^{6}$ Experimental lattice parameters [61]

${ }^{7}$ Calculated from experimental elastic constants [62]

${ }^{8}$ Calculated from experimental elastic constants [63] 
Table II. Experimental and predicted properties of $\mathrm{CaCO}_{3}$ phases

\begin{tabular}{|c|c|c|c|c|c|c|}
\hline & \multicolumn{3}{|c|}{ Calcite } & \multicolumn{3}{|c|}{ Aragonite } \\
\hline & Experimental & Calculated & $\Delta(\%)$ & Experimental & Calculated & $\Delta(\%)$ \\
\hline Elastic Constants (GPa) & & & & & & \\
\hline $\mathrm{C}_{11}$ & $145.7^{3}$ & 140.9 & -3.3 & $85.0^{1}$ & 89.9 & 6.5 \\
\hline $\mathrm{C}_{22}$ & & & & $159.6^{1}$ & 155.3 & -2.7 \\
\hline $\mathrm{C}_{33}$ & $85.3^{3}$ & 85.8 & 0.6 & $87.0^{1}$ & 104.2 & 20 \\
\hline $\mathrm{C}_{44}$ & $33.4^{3}$ & 33.4 & 0.0 & $42.7^{1}$ & 23.3 & -45 \\
\hline $\mathrm{C}_{55}$ & & & & $41.3^{1}$ & 36.7 & -11 \\
\hline $\mathrm{C}_{66}$ & & & & $25.6^{1}$ & 12.4 & -52 \\
\hline $\mathrm{C}_{12}$ & $55.9^{3}$ & 63.7 & 14 & $15.9^{1 *}$ & 48.0 & 202 \\
\hline $\mathrm{C}_{13}$ & $53.5^{3 *}$ & 62.6 & 17 & $36.6^{1} *$ & 55.9 & 53 \\
\hline $\mathrm{C}_{14}$ & $20.5^{3 *}$ & 19.5 & -4.9 & & & \\
\hline $\mathrm{C}_{23}$ & & & & $2.0^{1 *}$ & 54.7 & \\
\hline Static Dielectric & & & & & & \\
\hline$\varepsilon_{11}^{0}$ & $8.5^{4}$ & 9.28 & 9.2 & & 7.84 & \\
\hline$\varepsilon_{22}^{0}$ & & & & & 22.48 & \\
\hline$\varepsilon_{33}^{0}$ & $8.0^{4}$ & 8.30 & 3.7 & & 8.26 & \\
\hline High Frequency Dielectric & & & & & & \\
\hline$\varepsilon^{\infty}{ }_{11}$ & $2.75^{2}$ & 2.69 & -2.2 & $2.86^{2 *}$ & 3.05 & 6.6 \\
\hline$\varepsilon_{22}^{\infty}$ & & & & $2.82^{2 *}$ & 2.53 & -10 \\
\hline$\varepsilon_{33}^{\infty}$ & $2.21^{2}$ & 3.02 & 37 & $2.34^{2} *$ & 2.50 & 6.8 \\
\hline Vibrational frequencies $\left(\mathrm{cm}^{-1}\right)$ & & & & & & \\
\hline Asymmetric stretch (IR), $v_{3}$ & $1463^{5}$ & $\begin{array}{l}1465 \\
1573\end{array}$ & 3.8 & $1473^{5}$ & 1500 & 1.8 \\
\hline Symmetric C-O stretch (Raman), $v_{1}$ & $1088^{5}$ & $\begin{array}{l}1082 \\
1091\end{array}$ & -0.1 & $1086^{5}$ & 1124 & 3.5 \\
\hline Torsional bending (IR), $v_{2}$ & $881^{5}$ & $\begin{array}{l}878 \\
994\end{array}$ & 6.2 & $873^{5}$ & 781 & -11 \\
\hline Stretch/bend (IR), $v_{4}$ & $714^{5}$ & 612 & -14 & $705^{5}$ & 627 & -11 \\
\hline
\end{tabular}

*Properties not used in the potential fitting

${ }^{1}$ Experimental values from [63]

${ }^{2}$ Experimental values from [64]

${ }^{3}$ Experimental values from [62]

${ }^{4}$ Experimental values from [65]

${ }^{5}$ Experimental values averaged from [66] 
Table III. Point defect energies in calcite

\begin{tabular}{|lll|}
\hline Defect & Notation & Energy $(\mathrm{eV})$ \\
\hline Ca vacancy & $\mathrm{V}_{\mathrm{Ca}}$ & 20.53 \\
$\mathrm{CO}_{3}{ }^{2-}$ vacancy & $\mathrm{V}_{\mathrm{CO} 3}$ & 28.17 \\
Schottky pair $\mathrm{Ca}^{2+}-\mathrm{CO}_{3}{ }^{2-}$ & $\mathrm{V}_{\mathrm{Ca}}+\mathrm{V}_{\mathrm{CO} 3}$ & 43.38 \\
Cd substitution & $\mathrm{Cd}_{\mathrm{Ca}}$ & -1.20 \\
Mn substitution & $\mathrm{Mn}_{\mathrm{Ca}}$ & -2.01 \\
Fe substitution & $\mathrm{Fe}_{\mathrm{Ca}}$ & -2.56 \\
Zn substitution & $\mathrm{Zn}_{\mathrm{Ca}}$ & -2.76 \\
Co substitution & $\mathrm{Co}_{\mathrm{Ca}}$ & -2.79 \\
Ni substitution & $\mathrm{Ni}_{\mathrm{Ca}}$ & -3.35 \\
Mg substitution & $\mathrm{Mg}_{\mathrm{Ca}}$ & -2.70 \\
\hline
\end{tabular}


Table IV. Migration energies for cation diffusion in calcite

\begin{tabular}{|lll|}
\hline Diffusion cation & $\begin{array}{c}\text { Energy }(\mathrm{eV}) \\
|| \text { to } c \text {-axis }\end{array}$ & $\begin{array}{c}\text { Energy }(\mathrm{eV}) \\
\perp \text { to } c \text {-axis }\end{array}$ \\
\hline $\mathrm{Ca}^{2+}$ & 1.30 & 2.48 \\
$\mathrm{Mg}^{2+}$ & 1.00 & 1.95 \\
$\mathrm{Mn}^{2+}$ & 1.26 & 2.30 \\
\hline
\end{tabular}




\section{Figure Captions}

Figure 1. Hexagonal representation of the rhombohedral structure of calcite showing the alternating layering of calcium ions and carbonate groups along the $c$-axis of the unit cell.

Figure 2. Comparison of energy-optimized structures of the (1014) surface of calcite, dolomite, and magnesite as viewed normal to the surface; modified from [40,41]. The black lines for the carbonate groups and black dots for the metal ions represent the optimized bulk structure.

Figure 3. Variation of the torsion angle for the carbonate group as a function of layer depth for the dry and wet simulations of the (10ī) surface of calcite.

Figure 4. Crystal structure (hexagonal-based unit cell) of calcite indicating the possible diffusion paths for migration of a cation from an occupied site to a cation vacancy. Only atoms for the unit cell are noted; the black open box represents a cation vacancy or possible vacancy site. 
Figure 1

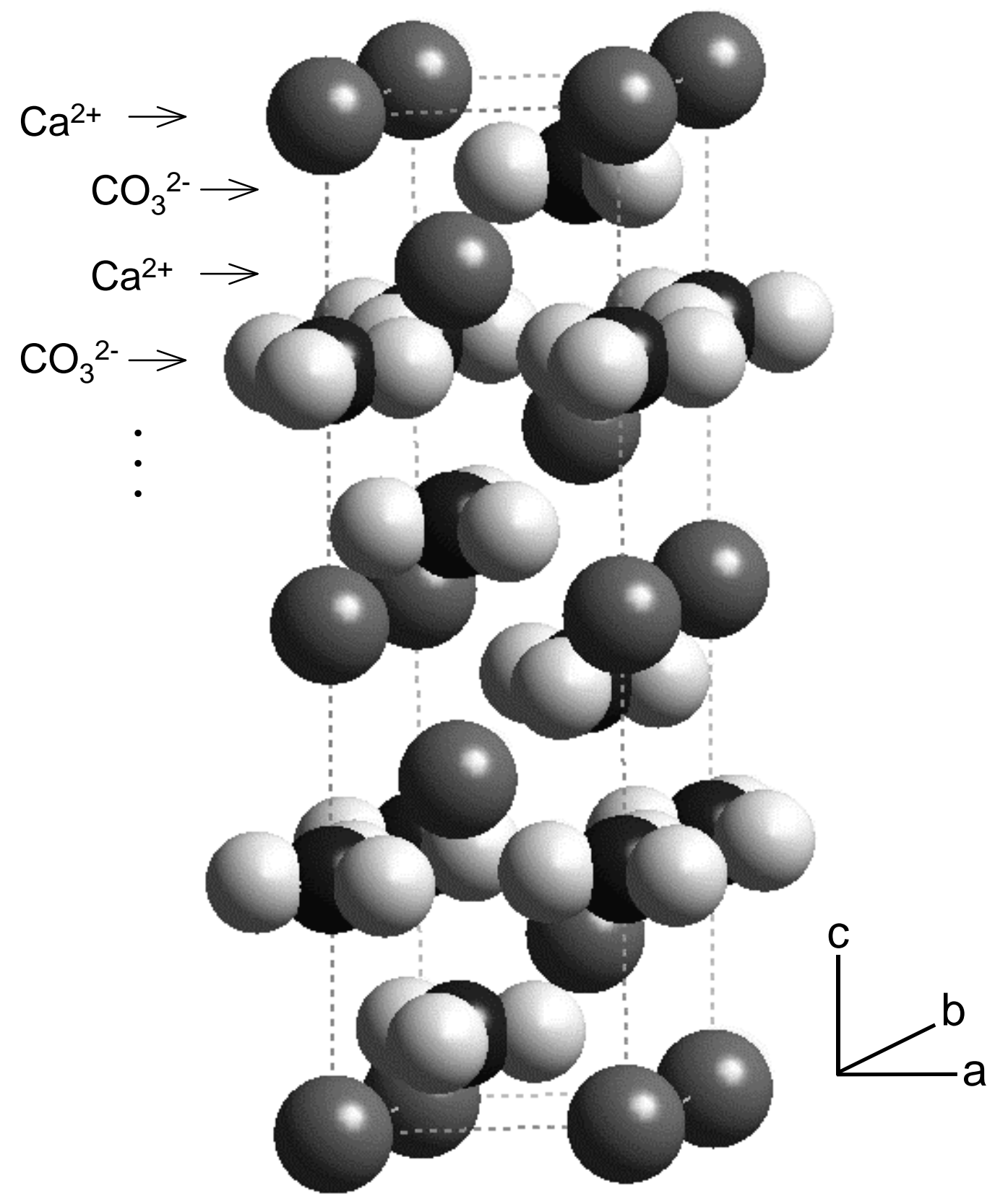




\section{Figure 2}

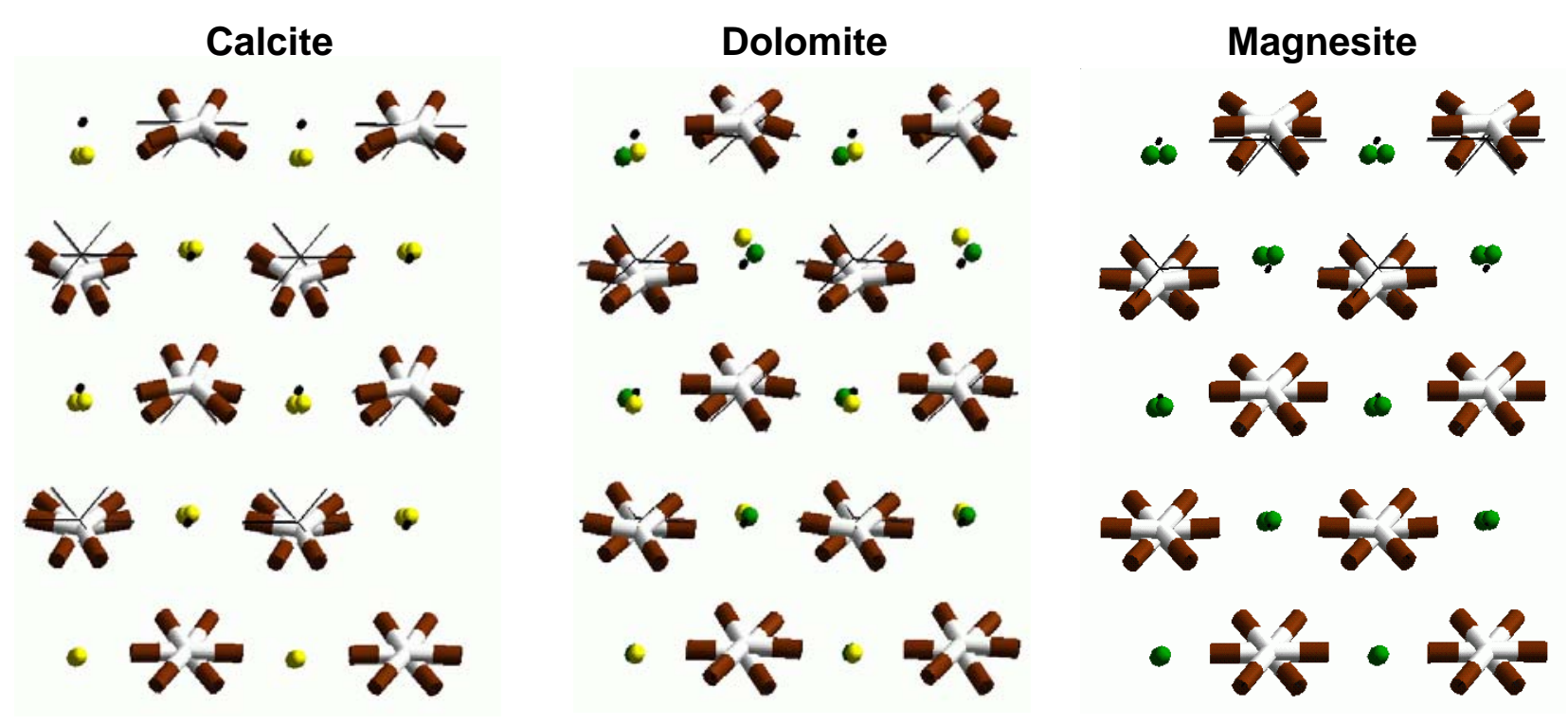


Figure 3

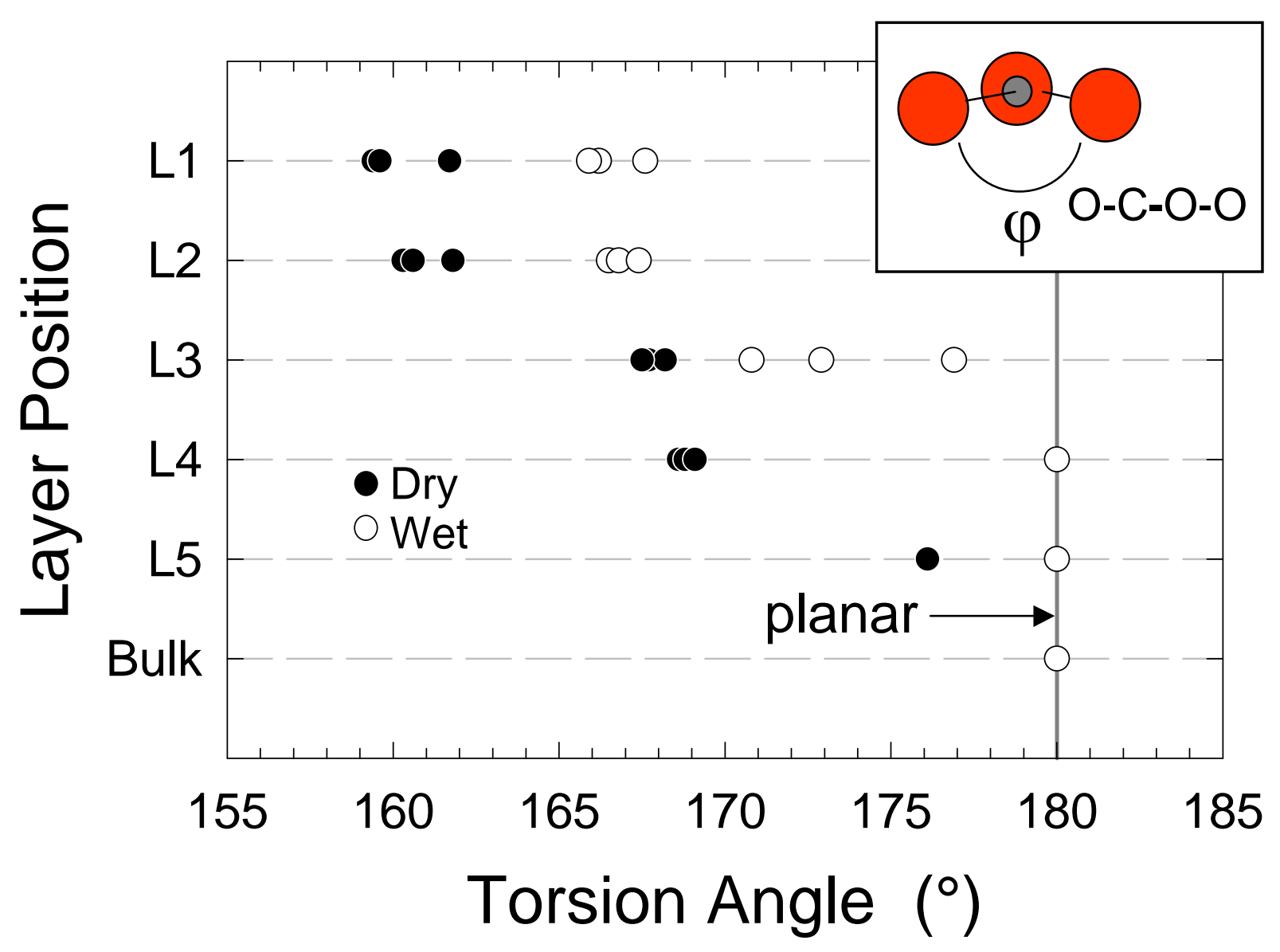


Figure 4

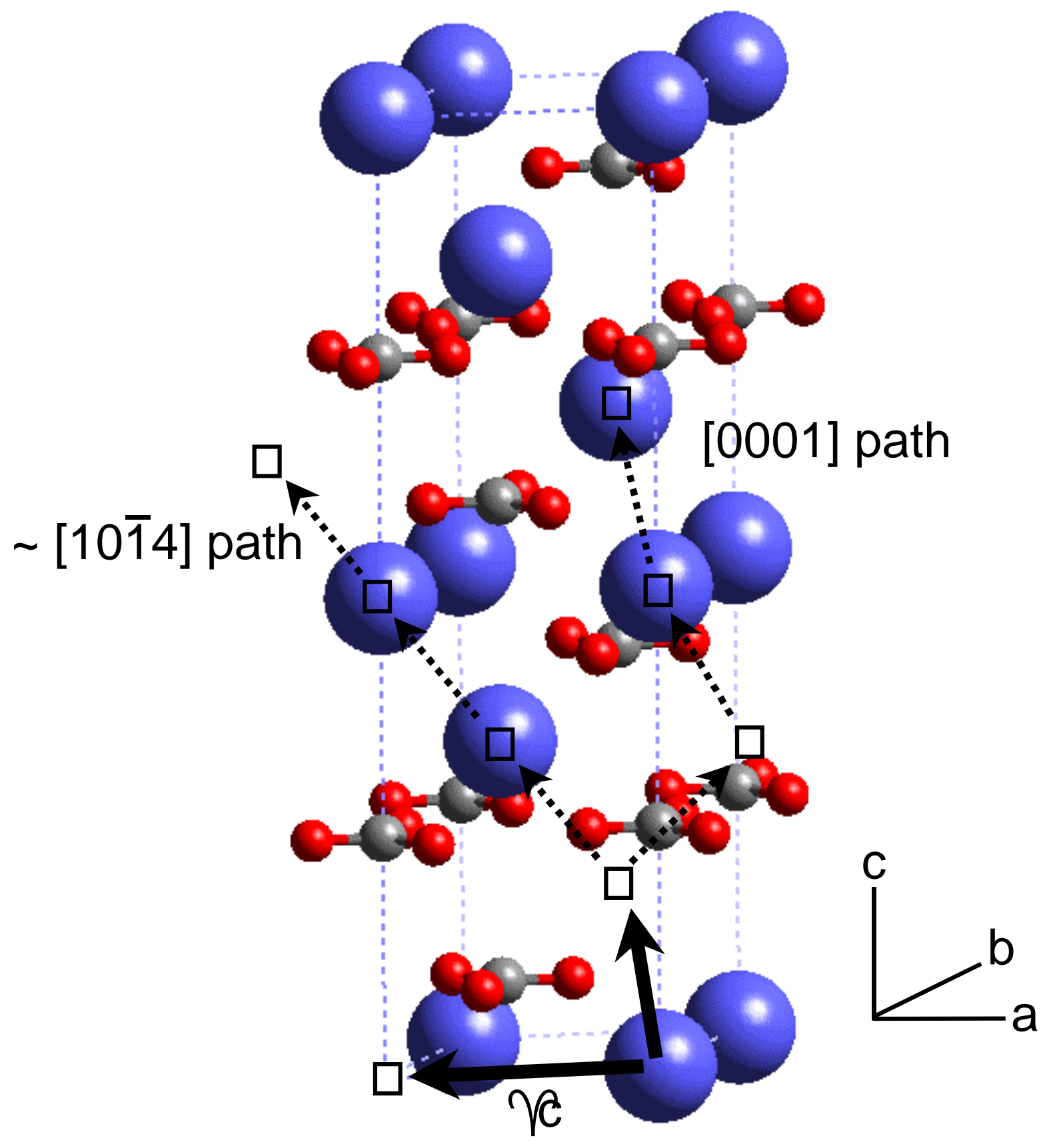

